Although primarily a record of one particular conference, the book appears to be aimed at people responsible for introducing new DNA services. Presentation is clear, with many diagrams and quality reproduction of autoradiographs. On the technical side, it provides a good source for key references (although these can be found in other recent comprehensive texts on genetic diseases), but fails as a reference for technical details through being already two years out of date. With these limitations each paper provides a good review up to 1986 of the clinical applications of molecular genetics in specific diseases, and may be of value to anyone wishing to read such a review in fields peripheral to their own immediate area of interest. Being very readable, the papers may also serve as a guide to staff involved in administrating molecular genetic services.

P W LunT

\section{The Neural Crest}

By Brian K Hall and Sven Hörstadius. (Pp 303; £30.00.) Oxford: Oxford Scientific Publications. 1988.

The neural crest has long fascinated biologists: firstly because of its transient nature, and secondly by virtue of the multitude of cells and organs, or parts of organs, which arise from it either directly or indirectly. This book reflects the long and continuing history of the search to unfold all the secrets which the neural crest holds, for it is not one book, but two separate monographs. There is a historical review and a summary of our present knowledge by Brian Hall, a Professor of Biology, followed by a reprint of Sven Hörstadius's classic work on the subject first published in 1950 .

The book shows that the neural crest is important in normal embryological development, in dysmorphology, and, perhaps surprisingly, in vertebrate evolution. No invertebrate possesses anything even remotely similar to the neural crest, while all vertebrates, with the possible exception of hagfishes, do. Consequently, the neural crest must have played a central role in the origin and evolution of vertebrates.

This book is probably more for biologists than clinicians, but that is not to say that those trained in medicine cannot delight in experimental biology and enjoy this book too. Hörstadius devoted his work almost entirely to an analysis of the amphibian neural crest: that of the newt, the frog, and the axolotl. Hall's section relates how most of what we know about the origin, function, and fate of the neural crest has been derived from ingenious transplantation, extirpation, and cell labelling experiments in a whole host of vertebrate species.

No critical comment is warranted on the Hörstadius monograph: it is a seminal work and it remains influential. Hall's contribution comprises 128 pages. The first three chapters are devoted to embryological origins, the neural crest, and the germ layer theory and our knowledge of the neural crest and its derivatives in hagfishes, lampreys, fish, amphibia, reptiles, birds, and mammals. Then come chapters on neural crest cell migration and differentiation. As long ago as 1868 , Wilhelm His first discovered the neural crest and noted that the cells moved away from their original location peripherally to form sensory ganglia. Today the use of monoclonal antibodies and other sophisticated techniques are showing that subpopulations of neural crest cells exist which are, at least, bipotential, and identifying the nature of the different microenvironments encountered by neural crest cells, which are major determinants of their migratory patterns and differentiation. The final two chapters are concerned with the neurocristopathies, syndromes, tumours, and dysmorphologies involving neural crest derived cells, and embryonic defects of the neural crest leading to malformations, in particular craniofacial defects. Together these two chapters occupy only 28 pages, which gives some indication as to the rather superficial nature of the monograph. It is not intended to be a detailed account: the author describes it as "a broad sweep", but one is left with a feeling of lack of depth. Nevertheless, it is well written and illustrated, and provides an interesting and easily readable overview and historical account of a topic which has wide ramifications.

Mary J Seller 